\title{
CLINICAL APPLICATION OF WHOLE EXOME SEQUENCING IN A CASE OF METASTASISED LOW GRADE SEROUS OVARIAN CANCER
}

Bruce Moran ${ }^{1,3}$, Michael Wilkinson¹, Sudipto Das², Darran O'Connor ${ }^{2}$, Jean Murphy³, Kieran Sheahan ${ }^{1,3}$ Cathy Kelly¹, Niall Mulligan¹, Brendan Loftus ${ }^{4,5}$, Donal Brennan ${ }^{1,5,6}$

(1): Ireland East Hospital Group, Mater Misericordiae University Hospital, Dublin 7. (2): Molecular and Cellular Therapeutics Lab., Royal College of Surgeons Ireland, Dublin 2. (3): Department of Histopathology, St. Vincent's University Hospital, Dublin 4.

(4): Core Sequencing Facility, Conway Institute, University College Dublin, Dublin 4. (5): School of Medicine, University College Dublin, Dublin 4. (6): Cancer Biology and Therapeutics Lab., Conway Institute, University College Dublin, Dublin

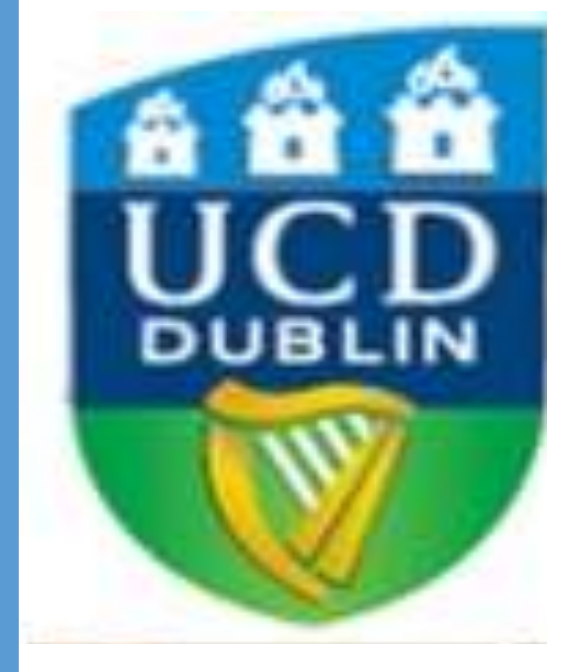

\section{Background}

Low grade Ovarian Serous cancer (LGOSC) is a recently described, rare histological subtype of ovarian cancer which is clinically and molecularly distinct from the four other main histological subtypes (high grade serous, clear cell, endometrioid and mucinous). Clinically it is distinguishable from high grade cancers by it's indolent course, resistance to chemotherapy and presentation at a much younger age. Genomically it is

characterized by mutations in KRAS, N-RAS and BRAF. The role of adjuvant therapy in patients with LGOSC is uncertain given it's relative chemo resistance. We report a case where whole exome sequencing identified a novel mutation allowing for specific targeting with chemotherapeutic agents.
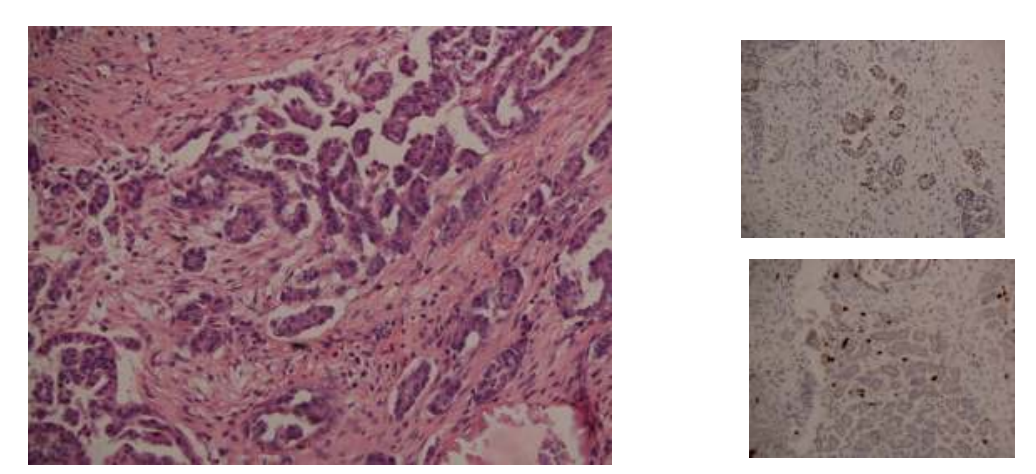
P53 wild type Low PI

Figure1: Histological representation of low grade serous ovarian cancer.

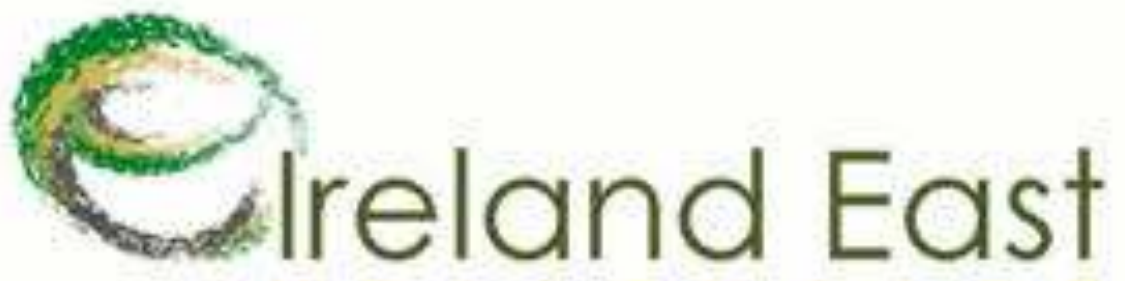

\section{Case History}

We report a 37 year old patient with LGOSC who underwent extensive cytoreductive surgery in December 2016. This was followed shortly after with 6 completed cycles of carboplatinum and paclitaxel. In November 2017 she experienced a recurrence in her chest and abdominal wall.

\section{Methods}

DNA was extracted from seven FFPE tumour samples (5 from primary surgery and 2 from recurrences) and whole blood. Exomes were sequenced using Illumina's NextSeq platform. Sequence data was trimmed, aligned and single nucleotide variants (SNVs) and copy number alterations (CNAs) were called. Single gene testing was performed for BRAF V600E, KRAS and ERFR. MSI status was confirmed using immunohistochemistry.

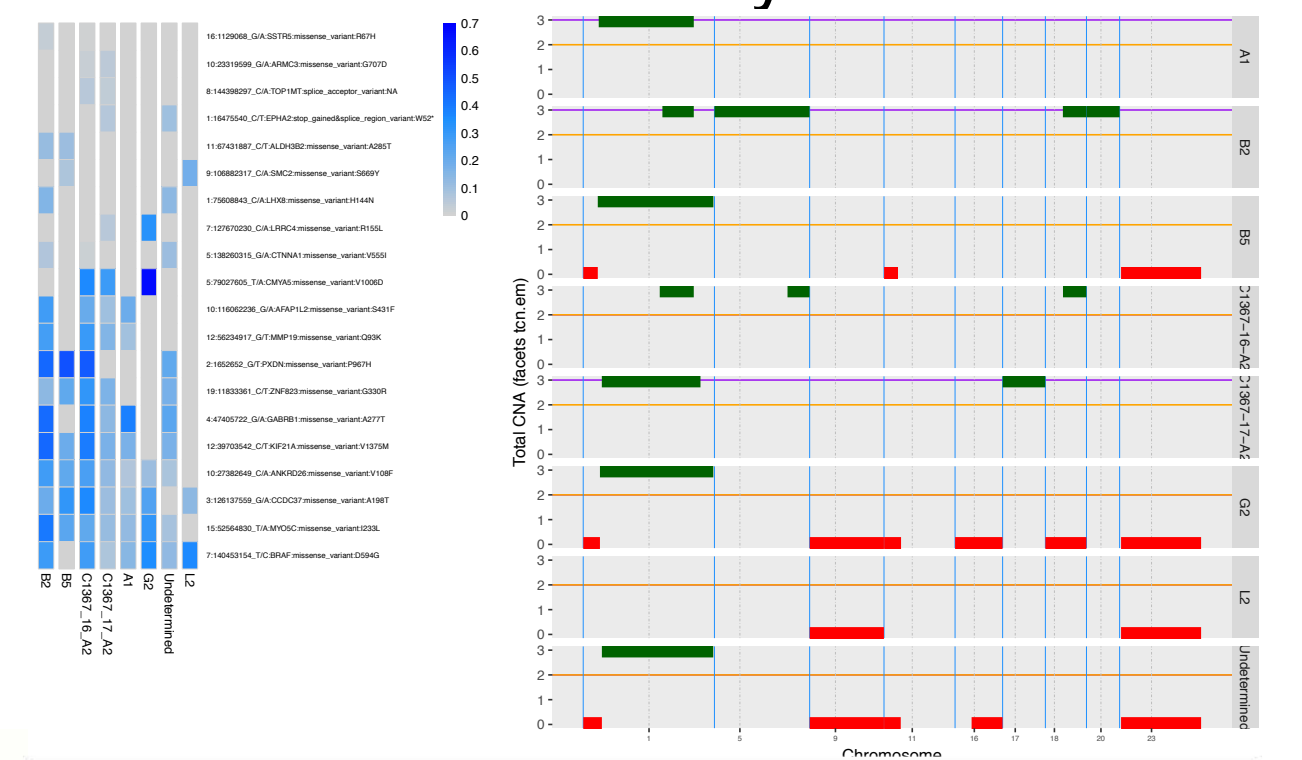

Translational/Interpretation

Class 3 'kinase-dead' BRAF variant, experimentally shown to activate MAPK, RAS by receptor tyrosine kinase signalling (see Znao eta al. 2017) Amplification includes NRAS

See Civic ab entry for BRAF 05946 Mutation testing (qPCR, SVUH histopathology lab) shows NRAS, KRAS, EGFR, BRAFV600E are wild-type

\section{Results}

The tumour had a low mutational burden. Among twenty protein-coding somatic SNVs reported. We identified a class 3 'kinase-dead' BRAF variant, D594G present in 6/7 tumour samples. This mutation is known to inhibit protein activity. Concordant nearwhole chromosome 1 amplification in both primary and recurrent samples, covering the NRAS protooncogene. Single gene testing confirmed wild type EGFR and KRAS and the tumour is microsatellite stable.

Discussion

Class 3 BRAF mutations increase MAPK activity by weakly binding CRAF which increases receptor tyrosine kinase mediated phosphorylation of RAS genes. This is particularly the case in RAS-activated tumours, likely here given NRAS amplification. As a result we have commenced this lady on MEK inhibitor (Trametinib) and she is awaiting restaging. These findings have not been previously described in LGSOC. 